\title{
Focus on neurodegenerative disease
}

\author{
Nature Neuroscience presents a special issue of recent advances on shared mechanisms underlying \\ neurodegenerative disease.
}

W

e tend to think of neurodegenerative diseases as separate clinical entities that target different brain regions with distinct pathology and symptoms. However, when considered at the genetic, molecular, or cellular level, certain players and patterns crop up again and again, such as early vascular dysfunction, the aggregation and spread of misfolded proteins, selective vulnerability of particular neurons, and activation of immune responses, to name but a few. Should we consider such pathological phenomena as arising from common mechanisms that play out across different brain regions and cell types, or as simply the same steps along a shared pathway to neurodegeneration? In this issue, we present a collection of Reviews describing recent advances in our understanding of neurodegenerative disease, the commonalities and differences between the major pathologies, and the gaps in our knowledge that still need to be addressed.

Gan et al (https://doi.org/10.1038/s41593018-0237-7) provide an overview of shared pathways disrupted across neurodegenerative diseases, from mitochondrial defects to dysfunctions in autophagic-lysosomal pathways and synaptic toxicity, as well as more recently emerging mechanisms, such as the roles of stress granule pathways and liquid-phase transitions in pathological protein aggregation.

Genomic analyses may provide valuable insights into the pathological processes of neurodegeneration. De Jager et al (https://doi. org/10.1038/s41593-018-0240-z) emphasize the need to complement classic case-control genetic association studies with wider population-based studies to better capture the full range of susceptibility variants and to determine how their interaction disrupts CNS function. The authors argue that the best chance at achieving these goals lies in collaborative efforts, working together to integrate multi-'omics' datasets to fully capture the impact of common diseaseassociated variants on the aging brain.

Some of the earliest pathological changes associated with neurodegeneration include neurovascular dysfunction. Zlokovic and colleagues (https://doi.org/10.1038/ s41593-018-0234-x) discuss the cellular pathways that regulate cerebral blood flow and blood-brain barrier integrity and how these go awry in neurodegenerative diseases, focusing on Alzheimer's disease. Future studies are needed to understand the role of neurovascular disruptions and whether this is a cause or a consequence of neurodegenerative disease.

Another hallmark of neurodegenerative diseases is the accumulation of misfolded proteins. Soto and Pritzkow (https:// doi.org/10.1038/s41593-018-0235-9) provide an overview of recent studies into protein misfolding and aggregation in neurodegeneration and consider the role of prion-like mechanisms and conformational strains in disease pathology. The notion that structural variations may underlie differences in aggregate toxicity or even disease progression has gained momentum in recent years, especially with the application of cryoelectron microscopy to better elucidate the structures of pathogenic proteins.

In a related piece, Jucker and Walker (https://doi.org/10.1038/s41593-018-02386) review the mechanisms underlying pathogenic protein propagation. The authors evoke the analogy of 'seed and soil' to discuss the importance of the host environment when it comes to the spread of proteins and pathology.

Neurodegenerative diseases can be characterized by the cell populations affected and the stereotypical fashion by which pathology develops across brain regions. Yet we are only just beginning to understand the mechanisms that underlie selective vulnerability. Duff and colleagues (https://doi.org/10.1038/s41593-0180221-2) propose that with advancing age, vulnerable neurons draw closer to the edge of 'catastrophic cliffs', requiring only a light shove to send a cell hurtling toward unchecked pathway dysregulation and degeneration. With the development of induced pluripotent stem cell modeling and single-cell profiling, we can now start to appreciate the biological processes that render certain cell types vulnerable to pathology while sparing others.

Of course, neurons are not the only cell types implicated in neurodegenerative disease. El Khoury and colleagues (https:// doi.org/10.1038/s41593-018-0242-x) provide an overview of the role of microglia in neurodegeneration, examining the ways in which microglial responses can shift from neuroprotective to deleterious over time. Considerable efforts are underway to profile microglia at the single-cell level and describe their heterogeneity in health and disease. The recent ability to differentiate microglialike cells from human induced pluripotent stem cells presents another promising tool, especially when combined with threedimensional organoids to better reproduce in vivo conditions.

While great steps have been made in human neuroimaging and stem cell modeling, a large proportion of neurodegenerative research still relies on animal models, especially rodent models. Dawson and colleagues (https://doi. org/10.1038/s41593-018-0236-8) discuss the current state of rodent models for some of the major neurodegenerative diseases. They consider ways to improve translatability in the wake of recent clinical trial failures and discuss strategies for the development of future models.

Although translating basic research into new treatments for neurodegenerative disease remains a major challenge, the field is well poised thanks to ongoing advances in animal and stem cell modeling, as well as in neuroimaging and single-cell profiling. Nature Neuroscience has already published a number of key advances in neurodegenerative research and looks forward to considering future studies. These include, but are not limited to, the next generation of human studies that leverage new tools and analysis platforms to interrogate the genetic underpinnings of these diseases, as well as future efforts to replicate strain-like features of human disease aggregates in cellular or animal models to better understand their role in disease pathology. We are keen to learn more about how stress granule dynamics are hijacked in neurodegenerative disease and about the cellular mechanisms that promote the translocation and spreading of aberrant misfolded proteins from one cell or brain region to another. We are also interested in more work demonstrating conservation of particular mechanisms across pathologically distinct neurodegenerative diseases, as well as therapeutic modalities aimed at targeting these pathways and showing benefit in various models.

We hope that these Reviews will inspire new research, and we look forward to publishing future advances in this important area of neuroscience.

Published online: 26 September 2018 https://doi.org/10.1038/s41593-018-0250-x 